\title{
A new gear fault diagnosis method based on improved local mean decomposition
}

\author{
Yu Wei ${ }^{1, a}$, Minqiang $\mathrm{Xu}^{2, \mathrm{~b}}$ and Yongbo $\mathrm{Li}^{3, \mathrm{c}}$ \\ ${ }^{1}$ Department of Astronautical Science and Mechanics, Harbin Institute of Technology (HIT), China \\ ${ }^{2}$ Department of Astronautical Science and Mechanics, Harbin Institute of Technology (HIT), China \\ ${ }^{3}$ Department of Astronautical Science and Mechanics, Harbin Institute of Technology (HIT), China \\ aweiyuhrbeu@126.com, bxumq@hit.edu.cn, ${ }^{c}$ liyongbo0532@126.com
}

Keywords: Feature extraction, Local mean decomposition (LMD), Fault diagnosis

Abstract. A new vibration feature extraction method based on improved local mean decomposition (LMD) is presented in this paper. Local mean decomposition is a novel adaptive time-frequency analysis method, which is widely used in rotating machinery fault diagnosis. However, traditional LMD decomposition results method is sensitive to noise. In order to eliminate influence of noise, Hermite-LMD is introduced. Firstly, the vibration signal is decomposed by Hermite-LMD method. Then, the fault frequency of gear is found through the envelope spectrum analysis of the first PF component. The effectiveness of the proposed method is verified by the simulation data and the practical gear fault diagnosis.

\section{Introduction}

Gear fault diagnosis has attracted considerable attention in recent years [1]. Among them, the vibration analysis method has been widely applied for diagnosing the rolling bearing fault due to its intrinsic merits of revealing bearing failure $[2,3]$.

Local mean decomposition (LMD) can self-adaptively decompose a complicated signal into a series of simple components defined as product functions (PFs), which is proposed by Jonathan S. Smith and successfully used in EEG field [4]. LMD can decompose a superimposed signal into a complex series of product functions (PFs) and a residual. Each PF component is equal to the product of an envelope signal $a(\mathrm{t})$ and a pure frequency modulation (FM) signal $s_{n}(\mathrm{t})$. Envelope signal is the product of the IA and IA is obtained by the pure FM signal. Therefore, LMD method can adaptively decompose any multi-component signal into several single-component AM-FM signals. It is generally accepted that the gear vibration signals measured by sensor represent multi-component, AM-FM feature, simultaneously a sum of mono-component can be obtained by applying LMD method. Hence, LMD is appropriate to process the gear vibration signals. However, moving average (MA) approach is performed in the original LMD algorithm, which has many problems. To overcome the drawbacks of original LMD, in this paper we adopt Hermite interpolation to construct the local mean function and envelope functions, called Hermite-LMD [5]. Therefore, Hermite-LMD is introduced to achieve the signal decomposition in this paper.

\section{Hermite-LMD algorithm}

LMD can decompose a superimposed signal into a complex series of PF components and a residual. Each PF component is equal to the product of an envelope signal $a(\mathrm{t})$ and a pure FM signal $s_{n}(\mathrm{t})$. It will not stop until the residual is a monotonic function. Envelope signal is the product of the IA and IF, which is calculated by the pure FM signal $s_{\text {in }}(\mathrm{t})$ in Eq. (1)

$$
f_{i}(t)=\frac{1}{2 \pi} \frac{d\left(\arccos _{i n}(t)\right)}{d t}
$$


In the traditional LMD method, the local mean function $m(\mathrm{t})$ and envelope estimation function $a(\mathrm{t})$ are constructed by using MA approach in the sifting process. Whereas the inappropriate sliding step sizes of MA has an adverse impact on the final calculation of IA and IF in the end. Therefore, the selection of the sliding step is a major problem. Since the cubic spline interpolation has good convergence and high smoothness, Hu. J introduced the cubic spline interpolation method to construct the local mean function $m(\mathrm{t})$ and envelope estimation function $a(\mathrm{t})$. However, the cubic spline interpolation is performed to construct the envelopes with outstanding overshoot and undershoot problems. Recently, the fitting method-cubic Hermite interpolation method is found optimum in the post treatments of CBSR. The cubic Hermite interpolation method is widely applied in engineering, which has a continuous first derivative property at the node. Therefore, the points are continue and smooth, and the line has an excellent conformal characteristic. Since gearbox vibration signal is non-stationary and nonlinear, the cubic Hermite interpolation is suitable for fitting envelope of the vibration signal. The calculation steps of Hermite-LMD algorithm is described as follows.

(1) Find out all the extreme points of the signal, and then fit upper envelope $E_{\text {up }}(\mathrm{t})$ and lower envelope $E_{\text {low }}(\mathrm{t})$ using cubic Hermite interpolation, respectively. Then local mean function $m(\mathrm{t})$ and envelope estimation function $a(\mathrm{t})$ can be obtained by Eq. (2) and Eq. (3).

$$
\begin{aligned}
& m(t)=\frac{E_{u p}(t)+E_{\text {low }}(t)}{2} \\
& a(t)=\frac{\left|E_{u p}(t)-E_{\text {low }}(t)\right|}{2}
\end{aligned}
$$

(2) After obtaining the local mean function $m(\mathrm{t})$ and envelope estimation function $a(\mathrm{t})$, Subtract the local mean function $m_{11}(\mathrm{t})$ from the original data $x(\mathrm{t})$, and then residual signal $h_{11}(\mathrm{t})$ can be written as

$$
h_{11}(\mathrm{t})=x(\mathrm{t})-m_{11}(\mathrm{t})
$$

$h_{11}(\mathrm{t})$ then divided by the amplitude function $a_{11}(\mathrm{t})$ to obtain $s_{11}(\mathrm{t})$ is given by

$s_{11}(\mathrm{t})=h_{11}(\mathrm{t}) / a_{11}(\mathrm{t})$

(3) Get the envelope $a_{12}(\mathrm{t})$ corresponding to $s_{11}(\mathrm{t})$, if the envelope function $a_{12}(\mathrm{t})=1$, stop the procedure and take $s_{12}(\mathrm{t})$ as the first purely frequency modulated signal. Otherwise, regard $s_{11}(\mathrm{t})$ as the original signal and repeat the step (2)-(5) continues $\mathrm{n}$ times until the envelope function $a_{1(\mathrm{n}+1)}(\mathrm{t})=1$, so $s_{\mathrm{n}}(\mathrm{t})$ is a pure frequency modulated signal, which can be expressed as Eq. (6)

$$
\left\{\begin{array}{c}
h_{11}(t)=x(t)-m_{11}(t) \\
h_{12}(t)=s_{11}(t)-m_{12}(t) \\
\mathrm{M} \\
h_{1 n}(t)=s_{1(n-1)}(t)-m_{1 n}(t)
\end{array}\right.
$$

(4) The corresponding envelope is obtained by Eq. (7)

$$
a_{1}(\mathrm{t})=a_{11}(\mathrm{t}) a_{12}(\mathrm{t}) \ldots a_{1 \mathrm{n}}(\mathrm{t})=\prod_{q=1}^{n} a_{1 q}
$$

The first product function $P F_{1}(\mathrm{t})$ can be constructed by the envelope function $a_{1}(\mathrm{t})$ and the purely frequency modulated signal $s_{1 \mathrm{n}}(\mathrm{t})$, which can be written as

$$
P F_{1}(\mathrm{t})=a_{1}(\mathrm{t}) s_{1 \mathrm{n}}(\mathrm{t})
$$

(5)This derived $P F_{1}(\mathrm{t})$ is then subtracted from the original time series $x(\mathrm{t})$, resulting in a new function $u_{1}(\mathrm{t})$. Regard $u_{1}(\mathrm{t})$ as a new data and repeat the above procedure $k$ times until $u_{\mathrm{k}}(\mathrm{t})$ is a constant or contains no more oscillations. 


$$
\left\{\begin{array}{c}
u_{1}(t)=x(t)-P F_{1}(t) \\
\vdots \\
u_{k}(t)=u_{k-1}(t)-P F_{k}(t)
\end{array}\right.
$$

Thus, the original signal can be reconstructed according to

$x(\mathrm{t})=\sum_{p=1}^{k} P F_{k}(t)+u_{k}(\mathrm{t})$

where $u_{\mathrm{k}}(\mathrm{t})$ is the residue and $k$ is the number of PF components

\section{Application to gear fault diagnosis}

In order to validate the effectiveness of the proposed method, the first experiment was conducted on a test rig of the transmission gearbox as shown in Fig.1 (a). The sample frequency is $10000 \mathrm{~Hz}$. The accelerometer is mounted on the base of floorstand, where the fault frequency is easy to find. The speed of the driving gear is set to be $1500 \mathrm{rpm}$. A wearing fault that one tooth of the driven gear was artificially removed has been input into the gearbox, and the defect size is $0.2 \mathrm{~mm}$, which is shown in Fig.1 (b).

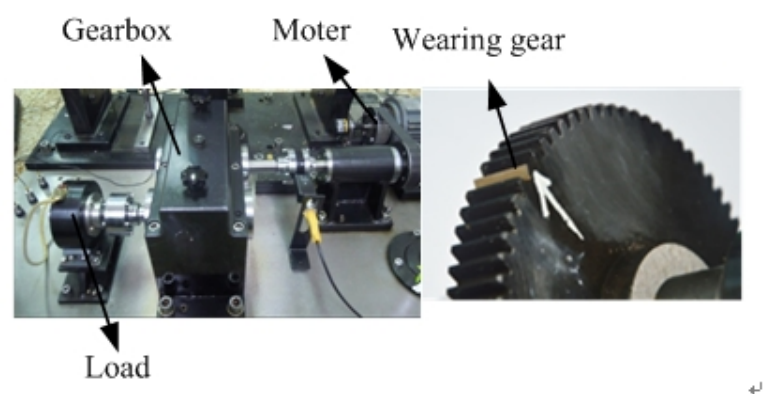

$\begin{array}{lll}\text { (a)The gearbox platform } & \text { (b) The wearing gear }\end{array}$

Fig.1. The fault experiment platform of mechanical vibration

In this experiment, the fault gear is the driven gear, so the fault frequency is $18.33 \mathrm{~Hz}$. The time domain of typical gear wearing fault vibration signal is illustrated in Fig.2.

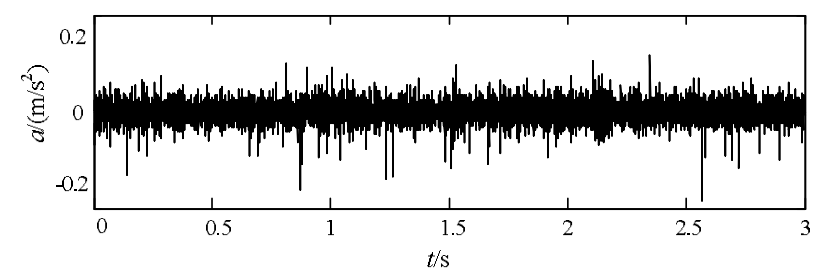

Fig.2. The acceleration signal of gear with slight wearing fault

To verify the advantage of the proposed method, a comparison between Hermite-LMD decomposition and traditional LMD is done. Since the PFs with different oscillatory modes are listed from high frequency to low frequency, and the fault information is mainly embedded in the high frequency, only the first four PFs are shown in Fig.3 and Fig.4, respectively.
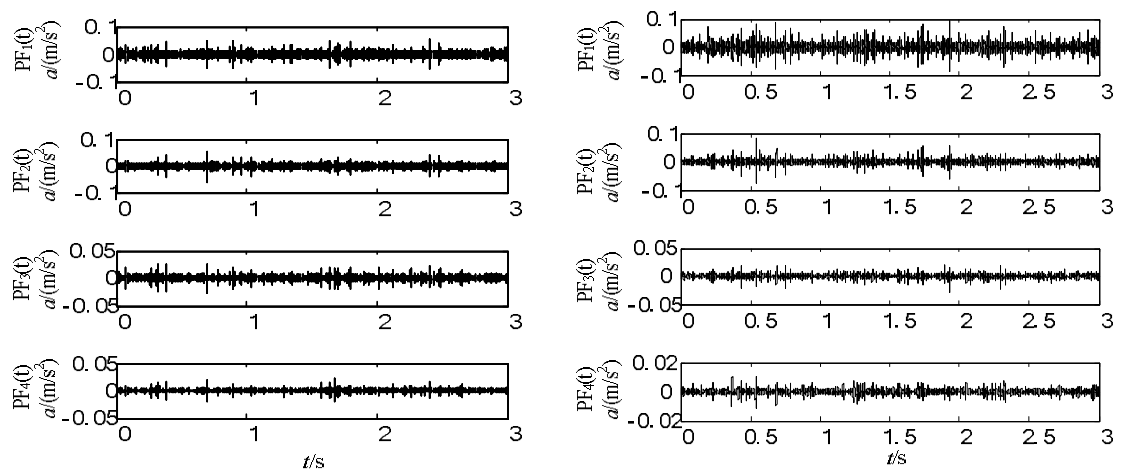

Fig.3. Traditional LMD decomposition results Fig.4. Hermite-LMD decomposition results

By observing the decomposition results of the two methods, the phenomenon of distortion can be easily found in Fig.3, while there is no such phenomenon occurring in the Fig.4. The comparisons verify the necessity of the signal pretreatment.

Secondly, the envelope spectrum analysis technique is applied to find the fault frequency ( $\mathrm{f}=18.33 \mathrm{~Hz}$ ). Since the fault 
information is mainly embedded in the high frequency, only the first PF component is selected to perform the envelope spectrum analyses. Fig.5 and Fig.6 give the amplitude spectrums of the $P F_{1}$ obtained by the two methods, respectively. Besides, for clearly comparison, the frequency-axis of all the figures in Figs is amplified by 10 times.

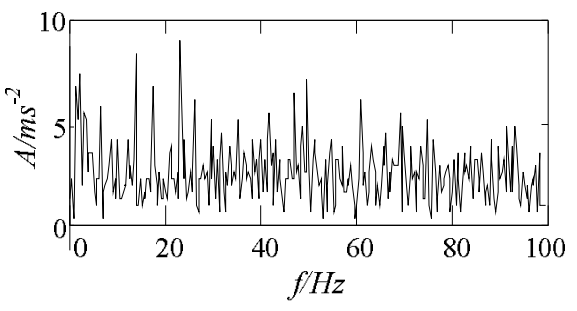

Fig.5. The amplitude spectrums of the $P F_{1}$ component of traditional LMD method.

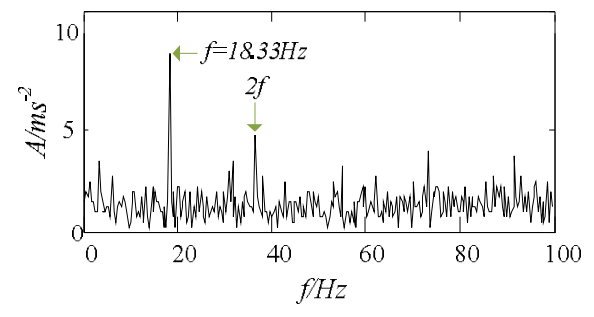

Fig.6. The amplitude spectrums of the $P F_{1}$ component of Hermite-LMD method.

For comparison purpose, the envelope spectrum difference of the $P F_{1}$ components derived from two methods should be discussed. As seen in Fig.5, we can't observe the accurate fault frequency, whereas, in Fig.6, obvious spectrum lines of the fault frequency and the double fault frequency can be found in the amplitude spectrums, avoiding the interference frequency. It is exactly consistent with the actual working condition. Hence, the comparisons show that Hermite-LMD method is feasible and effective in the gear fault diagnosis.

\section{Conclusions}

In this paper, a new feather extraction method based on Hermite-LMD is introduced. Firstly, the Hermite-LMD is performed to decompose the denoised signal. Compared with traditional LMD method, improved LMD can improve the accuracy decomposition results significantly. The effectiveness of the proposed method is validated by the application to the gearbox fault diagnosis in comparison with traditional LMD.

\section{Acknowledgements}

The work was supported by National Natural Science Foundation of China (NO. 11172078) and Important National Basic Research Program of China (973 Program-2012CB720003), and the authors are grateful to all the reviewers and the editor for their valuable comments.

\section{References}

[1] Q. He, Y. Liu and F. Kong. Machine fault signature analysis by midpoint-based empirical mode decomposition. Measurement Science and Technology, 2011.22(1): p.015702

[2]S. Olhede, Gao and A.T.Walden. The Hilbert spectrum via wavelet projections, Proc. R.Soc. Lond, Vol. 460 (2004), p. 955-975

[3] Y. Li, M. Xu, Y. Wei. An improvement EMD method based on the optimized rational Hermite interpolation approach and its application to gear fault diagnosis. Measurement, 2015, 63: p.330-345

[4] Smith, J.S. The local mean decomposition and its application to EEG perception data. Journal of the Royal Society Interface, 2005, 2(5): p.443-458

[5] Y. Li, M. Xu, Y. Wei. Application of CBSR and LMD in reciprocating compressor fault diagnosis. Journal of Vibroengineering, 2015, 17(1): p.203-215 